\title{
Beyond Hyperglycemia in Diabetes: Role of Statin Treatment on Thrombogenesis Triggered by Inflammation
}

\author{
Editorial to: "Impact of Statins on the Coagulation Status of Type 2 Diabetes Patients \\ Evaluated by a Novel Thrombin-Generations Assay" by P. Ferroni et al.
}

\section{Francesca Perego • Giovanni Davì}

Published online: 15 May 2012

(C) Springer Science+Business Media, LLC 2012

Type 2 diabetes mellitus (T2D) constitutes a growing global public health problem and its prevalence is estimated to increase during the next decades, especially in the developing countries. To date, $15 \%$ of the whole population is considered at high risk to develop T2D [1]. T2D is associated with a decrease of health-related quality of life and overall life expectancy, mainly due to an increased risk for cardiovascular disorders. Indeed, T2D is commonly associated with both microvascular and macrovascular complications [2]. Macrovascular complications manifest themselves as accelerated atherosclerosis, clinically resulting in premature coronary artery disease, increased risk of cerebrovascular disease, and severe peripheral vascular disease [2]. Patients with T2D have a a two- to four-fold increase in the risk of coronary artery disease (CAD) and patients with DM but without previous myocardial infarction (MI) carry the same level of risk for subsequent acute coronary events as non-diabetic patients with previous MI [3, 4] so that T2D has been defined a CAD equivalent.

T2D is associated with a prothrombotic state characterized by a number of changes in thrombotic and fibrinolytic coagulation factor level/activity, which together increase the risk of thrombus formation. Both glucose and insulin seem to play a

\author{
F. Perego \\ Internal Medicine III, Luigi Sacco Hospital, University of Milan, \\ Milan, Italy \\ G. Davì \\ Internal Medicine, University of Chieti, \\ Chieti, Italy \\ G. Davì $(\bowtie)$ \\ Center of Excellence on Aging, University of Chieti, \\ Via Colle dell'Ara, \\ 66013 Chieti, Italy \\ e-mail: gdavi@unich.it
}

role in the pathogenesis of this prothrombotic state [5]. In vitro induced thrombin generation is increased in platelet-rich plasma from diabetics compared with that from healthy subjects and a significant elevation of thrombin levels can be demonstrated in T2D patients in poor metabolic control when compared with well controlled patients [6]. Moreover, a significant correlation can be observed between improved glycemic control and blood thrombogenicity, as reflected by a reduction in ex-vivo thrombus formation in a Badimon perfusion chamber [7]. Improved glycemic control is the only significant predictor of a decrease in blood thrombogenicity, irrespective of treatment allocation [7]. Enhanced levels of prothrombin fragment $1+2(\mathrm{~F} 1+2)$ and thrombin-antithrombin complexes (TAT) have been shown in T2D patients in comparison with healthy subjects [8].

A hypercoagulable state, detected by thrombin generation tests, was reported in patients with $\mathrm{T} 2 \mathrm{D}$, explaining, at least partially, the high incidence of vascular events in these patients [9].

Platelets of patients with DM are characterized by dysregulation of several signaling pathways, leading to an hyperreactive phenotype with enhanced adhesion, aggregation, and activation [10]. Chronic hyperglycemia has been clearly identified as a causal factor for in vivo platelet activation [11]. Our group firstly demonstrated enhanced thromboxane (TX) biosynthesis in T2D, providing evidence for its platelet origin. Moreover, tight metabolic control led to reduction of TX metabolite urinary levels [11]. Interestingly, inflammatory mediators (such as CD40L) derived from platelets expand the functional repertoire of platelets from players of hemostasis and thrombosis to powerful amplifiers of inflammation by promoting the release of cytokines and chemokines, cell activation and cell-cell interactions [12].

T2D is frequently associated with a dyslipidemic state that may accelerate the progression of atherosclerotic 
disease and the development of cardiovascular disease (CVD) [1]. Similarly to T2D, dyslipidemia has been associated with altered coagulation leading to an increased risk of hypercoagulability through mechanisms linked to insulin resistance [7]. However, the mechanisms linking dyslipidemia, hyperglycemia and hypercoagulability are complex and to date have been only partially delineated (Fig. 1).

In this issue of Cardiovascular Drugs and Therapy, Ferroni et al. report on the performance of a novel activated protein $\mathrm{C}$ (APC)-dependent thrombin-generation assay in a case-control study in an unselected population of patients with longstanding T2D representative of a general practice cohort [13]. In order to minimize the contribution of known $\mathrm{CV}$ risk factors on the coagulation status, the authors select individuals with risk factors such as hypertension and dyslipidemia, or overt CVD in the control group. Nonetheless, the proportion of these diseases were different in the two groups; moreover, in the control group, individuals treated with aspirin and statins were also included. Thus, the interpretation of results on this peculiar population suggests caution limiting the external validity of the study. Taking into consideration these limitations, the results presented add further evidence on the occurrence of a procoagulant status in patients with T2D. This is particularly interesting, since the majority of case-control studies includes healthy individuals without atherothrombotic disorders or vascular disease and, thus, might be potentially biased by the occurrence of T2D complications or comorbidities.

As a corollary finding, Ferroni et al. report a significant association between the procoagulant status of T2D patients and the occurrence of in vivo platelet activation, as already described in diabetes [14]. They show an increase of the soluble form of CD40 ligand (sCD40L) in patients with $\mathrm{T} 2 \mathrm{D}$, confirming previously published observations in both type 1 and 2 diabetes [15-17].

The major finding of the study by Ferroni et al., however, is the demonstration of a significant association between coagulation abnormalities and statin treatment in T2D. It would be interesting to compare T2D/statins + vs control/ statins + individuals in order to assess the extent of the difference of statins on the coagulation status. Clinical and

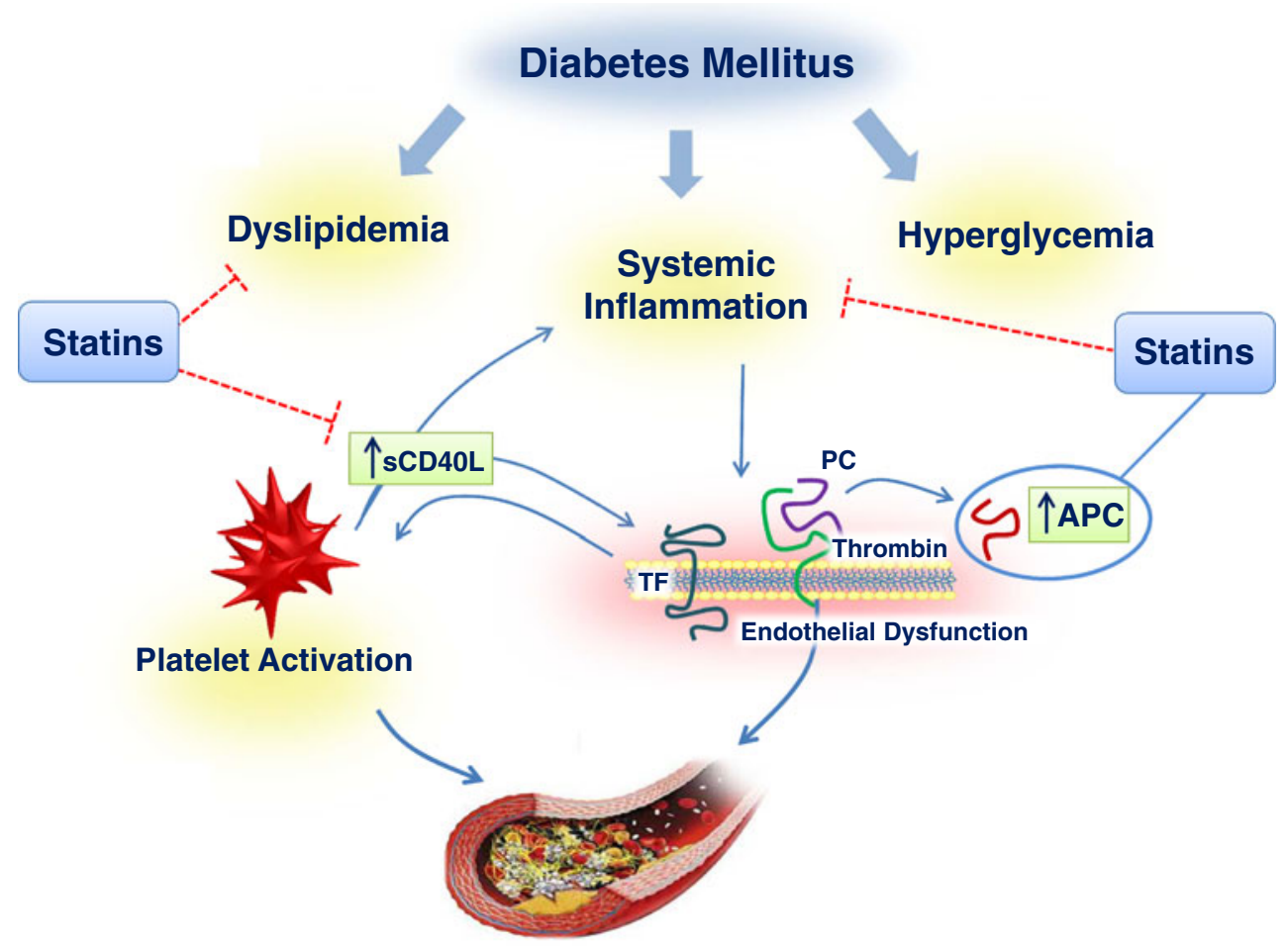

Atherothrombosis

Fig. 1 Role of statin treatment on thrombogenesis triggered by inflammation in T2D. T2D, characterized by chronic hyperglycemia, is frequently associated with a dyslipidemic state. In both hyperglycemia and dyslipidemia, it has been clearly identified a dysregulation of several platelets signaling pathways, through mechanisms linked to insulin resistance, leading to an hyperreactive platelet phenotype with enhanced platelet adhesion, aggregation, and activation. The release of platelet-derived inflammatory mediators, such as $\mathrm{SCD} 40 \mathrm{~L}$, expands the functional repertoire of platelets from players of hemostasis and thrombosis to powerful amplifiers of inflammation, thus promoting the increase of APC. These mechanisms contribute to the endothelial dysfunction that favors the atheromatous plaque formation. Statins can interfere at different levels of the inflammatory process, decreasing cholesterol levels, platelet activation and their release of mediators of inflammation. Taken together, these mechanisms may lead to stabilization of the atherosclerotic plaque as well as modulation of the prothrombotic status 
experimental studies have well established the pleiotropic effects of statins by showing their ability to reduce the inflammatory burden, to modulate endothelial function and the risk of thrombosis, thus reducing the incidence of acute vascular events [18]. Statins have been shown to lead to a significant downregulation of the blood coagulation cascade, independently of changes in lipid profile [18].

The findings reported by Ferroni et al., indeed, fit well into the current view of the antithrombotic effects of statins. In fact, a recent meta-analysis, in patients with diabetes taking statin therapy, demonstrated a proportional $9 \%$ decrease in all-cause mortality per $\mathrm{mmol} / \mathrm{L}$ reduction in $\mathrm{LDL}$, and a proportional $21 \%$ reduction in major vascular events per $\mathrm{mmol} / \mathrm{L}$ decrease in LDL, for patients on statin treatment. The effects of statin therapy were seen irrespective of baseline CVD risk, suggesting the use of an HMG-CoA reductase inhibitor in most patients with diabetes [19]. Moreover, the results presented by Ferroni et al. are consistent with current treatment recommendations. In fact, in patients with atherogenic dyslipidemia and T2D, lifestyle modifications and statin therapy are recommended regardless of baseline LDL for those with overt CVD [20]. At times, for individuals in primary prevention aged $>40$ years with one or more CVD risk factors the use not only of statin therapy but also of aspirin, both involved in the reduction of different prothrombothic pathways, is a topic of debate. On the basis of the results of three recent meta-analyses [21-23], no clear evidence support the routine use of lowdose aspirin for primary prevention of CVD in general population. The risk of adverse effects such as bleeding and benefit ratio should be weighed in every subject though it is reasonable to speculate that moderate-high risk patients for CVD with low bleeding risk can benefit from aspirin use. Unfortunately, current data support only a modest mortality benefit for statin primary prevention.

The data presented by Ferroni et al. confirm the role of statin in reducing hypercoagulability in patients with long standing T2D. They propose a standardized APC-based thrombin generation assay that should be tested as a prognostic marker in prospective (randomized) studies considering clinical relevant outcomes. From a clinical point of view it could be interesting to compare this novel assay to other available markers (F1+2, TAT) [5] to establish (if possible) a reference standard for the detection of the pro-thrombotic status.

These results are another step towards the development of new therapeutic strategies even in the early phase of atherothrombotic diseases. Atherothrombosis is a leading cause of death in patients with DM. The mechanisms leading to diabetic vascular disease are intimately related to altered glucose metabolism as well as to low-grade systemic inflammation, and prothrombotic coagulation abnormalities. Future researches should take into account these specific disease-based mechanisms, in order to decrease the accelerated atherosclerosis and the consequent thrombotic burden in diabetic patients.

Acknowledgments The Authors gratefully acknowledge the expert assistance of Sara La Barba, and Nicole Santoro.

Conflict of interest statement The authors have no conflict of interest to disclose.

\section{References}

1. Adeghate E, Schattner P, Dunn E. An update on the etiology and epidemiology of diabetes mellitus. Ann N Y Acad Sci. 2006;1084:1-29.

2. Luscher TF, Creager MA, Beckman JA, Cosentino F. Diabetes and vascular disease: pathophysiology, clinical consequences, and medical therapy. Circulation. 2003;108:1655-61.

3. Haffner SM, Lehto S, Ronnemaa T, Pyorala K, Laakso M. Mortality from coronary heart disease in subjects with type 2 diabetes and in nondiabetic subjects with and without prior myocardial infarction. $\mathrm{N}$ Engl J Med. 1998;339:229-34.

4. Beckman JA, Creager MA, Libby P. Diabetes and atherosclerosis: epidemiology, pathophysiology, and management. JAMA. 2002;287:2570-81.

5. Vazzana N, Ranalli P, Cuccurullo C, Davi G. Diabetes mellitus and thrombosis. Thromb Res. 2012;129:371-7.

6. Lemkes BA, Hermanides J, Devries JH, Holleman F, Meijers JC, Hoekstra JB. Hyperglycemia: a prothrombotic factor? J Thromb Haemost. 2010;8:1663-9.

7. Osende JI, Badimon JJ, Fuster V, et al. Blood thrombogenicity in type 2 diabetes mellitus patients is associated with glycemic control. J Am Coll Cardiol. 2001;38:1307-12.

8. Davi G, Gennaro F, Spatola A, et al. Thrombin-antithrombin III complexes in type II diabetes mellitus. J Diabetes Complicat. 1992;6:7-11.

9. Tripodi A, Branchi A, Chantarangkul V, et al. Hypercoagulability in patients with type 2 diabetes mellitus detected by a thrombin generation assay. J Thromb Thrombolysis. 2011;31:165-72.

10. Davi G, Patrono C. Platelet activation and atherothrombosis. N Engl J Med. 2007;357:2482-94.

11. Davi G, Catalano I, Averna M, et al. Thromboxane biosynthesis and platelet function in type II diabetes mellitus. N Engl J Med. 1990;322:1769-74.

12. Santilli F, Basili S, Ferroni P, Davi G. CD40/CD40L system and vascular disease. Intern Emerg Med. 2007;2:256-68.

13. Ferroni P, Della Morte M, Pileggi A, et al. Impact of statins on the coagulation status of type 2 diabetes patients evaluated by a novel thrombin-generation assay. Cardiovasc Drugs Ther. 2012;26: this issue.

14. De Cristofaro R, Rocca B, Vitacolonna E, et al. Lipid and protein oxidation contribute to a prothrombotic state in patients with type 2 diabetes mellitus. J Thromb Haemost. 2003;1:250-6.

15. Santilli F, Davi G, Consoli A, et al. Thromboxane-dependent CD40 ligand release in type 2 diabetes mellitus. J Am Coll Cardiol. 2006;47:391-7.

16. Lim HS, Blann AD, Lip GY. Soluble CD40 ligand, soluble Pselectin, interleukin-6, and tissue factor in diabetes mellitus: relationships to cardiovascular disease and risk factor intervention. Circulation. 2004; 109:2524-8.

17. Varo N, Vicent D, Libby P, et al. Elevated plasma levels of the atherogenic mediator soluble CD40 ligand in diabetic patients: a novel target of thiazolidinediones. Circulation. 2003;107:2664-9.

18. Undas A, Brummel-Ziedins KE, Mann KG. Statins and blood coagulation. Arterioscler Thromb Vasc Biol. 2005;25:287-94. 
19. Kearney PM, Blackwell L, Collins R, et al. Efficacy of cholesterollowering therapy in 18,686 people with diabetes in 14 randomised trials of statins: a meta-analysis. Lancet. 2008;371:117-25.

20. Kumar A, Singh V. Atherogenic dyslipidemia and diabetes mellitus: what's new in the management arena? Vasc Health Risk Manag. 2010;6:665-9.

21. Berger JS, Lala A, Krantz MJ, Baker GS, Hiatt WR. Aspirin for the prevention of cardiovascular events in patients without clinical cardiovascular disease: a meta-analysis of randomized trials. Am Heart J. 2011;162:115-24.

22. Raju N, Sobieraj-Teague M, Hirsh J, O’Donnell M, Eikelboom J. Effect of aspirin on mortality in the primary prevention of cardiovascular disease. Am J Med. 2011;124:621-9.

23. Seshasai SR, Wijesuriya S, Sivakumaran R, et al. Effect of aspirin on vascular and nonvascular outcomes: meta-analysis of randomized controlled trials. Arch Intern Med. 2012;172:209-16. 